\title{
CONTACT TWISTOR SPACES AND ALMOST CONTACT METRIC STRUCTURES
}

\author{
JOHANN DAVIDOV, CHRISTIAN L. YANKOV
}

\begin{abstract}
The notions of a twistor space of a contact manifold and a contact connection on such a manifold have been introduced by L. Vezzoni as extensions of the corresponding notions in the case of a symplectic manifold. Given a contact connection on a contact manifold one can define an almost $C R$-structure on its twistor space and Vezzoni has found the integrability condition for this structure. In the present paper it is observed that the $C R$-structure is induced by an almost contact metric structure. The main goal of the paper is to obtain necessary and sufficient conditions for normality of this structure in terms of the curvature of the given contact connection. Illustrating examples are discussed at the end of the paper.
\end{abstract}

Mathematics Subject Classification (2010): 53C28; 53D15

Key words: twistor spaces, almost contact metric structures.

\section{INTRODUCTION}

Let $M$ be a contact manifold with contact form $\alpha$ and contact distribution $\mathcal{D}$. The restriction of $d \alpha$ to $\mathcal{D}$ is a symplectic form and L. Vezzoni [10 has defined the contact twistor space of $M$ as the bundle over $M$ whose fiber at any point $p \in M$ consists of all complex structures on $\mathcal{D}_{p}$, the fibre of $\mathcal{D}$ at $p$, compatible with the symplectic form $d \alpha \mid \mathcal{D}$. He has also introduced the notion of a contact connection on $M$ and showed that, given such a connection $\nabla$, one can define a natural almost $C R$ structure on the contact twistor space of $M$ in a way that resembles the standard twistor construction. Vezzoni has found the integrability condition for this almost $C R$-structure in terms of the curvature of the connection $\nabla$.

In this note we observe that the $C R$-structure considered in $[10$ is induced by an almost contact metric structure on the contact twistor space of $M$. As usual in twistor theory, one can define one more almost contact metric structure and the main purpose of this paper is to discuss the normality of these almost contact metric structures. Recall that normality is an important property of an almost contact manifold $N$, which means that the product manifold $N \times S^{1}$ is a complex manifold with the complex structure induced by the almost contact one (cf., for example, [1]). As one can expect, the integrability condition for the first almost contact metric structure can be expressed in terms of the curvature of the connection $\nabla$, while the second one is never normal. Considering the two induced $C R$-structures, we reprove the Vezzoni integrability result for the first one and show that the second $C R$-structure is never integrable. Examples illustrating the obtained results are discussed in the last section of the paper.

The first named author is partially supported by the National Science Fund, Ministry of Education and Science of Bulgaria under contract DFNI-I 02/14. 


\section{Preliminaries}

2.1. Compatible complex structures on a symplectic vector space. Let $\mathcal{D}$ be a $2 n$-dimensional real vector space endowed with a non-degenerate skewsymmetric 2 -form $\omega$. Denote by $\mathcal{Z}=\mathcal{Z}(\mathcal{D}, \omega)$ the set of all complex structures $J$ on the vector space $\mathcal{D}$ compatible with the form $\omega$, i.e. such that $\omega(J x, J y)=\omega(x, y)$ and $\omega(x, J x)>0$ if $x \neq 0$. This set is a submanifold of the symplectic Lie algebra

$$
\operatorname{sp}(\omega)=\{S \in \operatorname{End}(\mathcal{D}): \omega(S x, y)+\omega(x, S y)=0, x, y \in \mathcal{D}\} .
$$

The tangent space $T_{J} \mathcal{Z}$ (considered as a subspace of $s p(\omega)$ ) is

$$
T_{J} \mathcal{Z}=\{V \in \operatorname{End}(\mathcal{D}): V J+J V=0, \omega(V x, y)+\omega(x, V y)=0\} .
$$

The smooth manifold $\mathcal{Z}$ admits a natural almost complex structure $\mathcal{J}$ defined by

$$
\mathcal{J} V=J V \text { for } V \in T_{J} \mathcal{Z}
$$

Every $J \in \mathcal{Z}$ is an orthogonal transformation of $\mathcal{D}$ with respect to the Euclidean metric $g_{J}(x, y)=\omega(x, J y)$. If $E_{1}, \ldots, E_{2 n}$ is a $g_{J}$-orthonormal basis such that $J E_{i}=E_{i+n}, i=1, \ldots, n$, then

$$
\omega\left(E_{i}, E_{j}\right)=\omega\left(E_{i+n}, E_{j+n}\right)=0, \quad \omega\left(E_{i}, E_{j+n}\right)=\delta_{i j} .
$$

A basis that satisfies the latter identities is called symplectic. Vice versa, given a symplectic basis $E_{1}, \ldots, E_{2 n}$, define a complex structure $J$ on the vector space $\mathcal{D}$ setting $J E_{i}=E_{i+n}, J E_{i+n}=-E_{i}, i=1, \ldots, n$. Then $J \in \mathcal{Z}$ and the symplectic basis is $g_{J}$-orthonormal.

The metric $g_{J}$ induces a metric $G_{J}$ on the vector space $\operatorname{End}(\mathcal{D})$,

$$
G_{J}(A, B)=\operatorname{Trace}\left\{\mathcal{D} \ni x \rightarrow g_{J}(A x, B x)\right\}, \quad A, B \in \operatorname{End}(\mathcal{D}) .
$$

Then $\mathcal{Z} \ni J \rightarrow G_{J} \mid T_{J} \mathcal{Z}$ is a (smooth) Riemannian metric on the manifold $\mathcal{Z}$ compatible with the almost complex structure $\mathcal{J}$.

Let $J \in \mathcal{Z}$ and let $E_{\alpha}, \alpha=1, \ldots, 2 n$, be an orthonormal basis of $\mathcal{D}$ with respect to the metric $g_{J}$ such that $J E_{i}=E_{i+n}, i=1, \ldots, n$. Define a basis $L_{\alpha \beta}$ of the vector space $\operatorname{End}(\mathcal{D})$ by

$$
L_{\alpha \beta} E_{\gamma}=\delta_{\alpha \gamma} E_{\beta}, \quad \alpha, \beta, \gamma=1, \ldots, 2 n .
$$

This basis is orthonormal with respect to the metric $G_{J}$ on $\operatorname{End}(\mathcal{D})$ induced by $g_{J}$. Set

$$
\begin{gathered}
V_{i j}=\frac{1}{2}\left(L_{i+n, j}+L_{j+n, i}+L_{i, j+n}+L_{j, i+n}\right), \quad i \neq j, \quad i, j=1, \ldots, n, \\
V_{i i}=\frac{1}{\sqrt{2}}\left(L_{i+n, i}+L_{i, i+n}\right), \quad i=1, \ldots, n .
\end{gathered}
$$

These endomorphisms of $\mathcal{D}$ are orthonormal, lie in $T_{J} \mathcal{Z}$ and

$$
\begin{gathered}
\mathcal{J} V_{i j}=\frac{1}{2}\left(L_{i+n, j+n}+L_{j+n, i+n}-L_{i, j}-L_{j, i}\right), \quad i \neq j \quad i, j=1, \ldots, n, \\
\mathcal{J} V_{i i}=\frac{1}{\sqrt{2}}\left(L_{i+n, i+n}-L_{i, i}\right), \quad i=1, \ldots, n .
\end{gathered}
$$

Thus $\left\{V_{i j}, \mathcal{J} V_{i j}: 1 \leq i \leq j \leq n\right\}$ is a $G_{J}$-orthonormal basis of $T_{J} \mathcal{Z}$.

Denote by $\operatorname{symm}(\omega)$ the set of endomorphisms $S$ of $\mathcal{D}$ that are $\omega$-symmetric, i.e. $\omega(S x, y)=\omega(x, S y)$. Then

$$
\operatorname{End}(\mathcal{D})=\operatorname{sp}(\omega) \oplus \operatorname{symm}(\omega) .
$$


Given $A \in \operatorname{End}(\mathcal{D})$ define an endomorphism $A^{*}$ of $\mathcal{D}$ by

$$
\omega\left(A^{*} x, y\right)=\omega(x, A y) \text {. }
$$

Then $\check{A}=\frac{1}{2}\left(A-A^{*}\right)$ and $\hat{A}=\frac{1}{2}\left(A+A^{*}\right)$ are the projections of $A$ onto $\operatorname{sp}(\omega)$ and $\operatorname{symm}(\omega)$, respectively.

For every $J \in \mathcal{Z}$, we also have the direct sum decomposition

$$
s p(\omega)=T_{J} \mathcal{Z} \oplus\{S \in \operatorname{sp}(\omega): J S-S J=0\} .
$$

The projection of an endomorphism $U \in s p(\omega)$ to $T_{J} \mathcal{Z}$ is given by $\operatorname{pr}_{J}(U)=$ $\frac{1}{2}(U+J U J)$. Thus

$$
\operatorname{pr}_{J}(A)=\frac{1}{2}(\check{A}+J \check{A} J) .
$$

is the projection of $A \in \operatorname{End}(\mathcal{D})$ to $T_{J} \mathcal{Z}$ with respect to the decomposition

$$
\operatorname{End}(\mathcal{D})=T_{J} \mathcal{Z} \oplus\{S \in \operatorname{sp}(\omega): J S-S J=0\} \oplus \operatorname{symm}(\omega) .
$$

Let $U \in T_{J} \mathcal{Z}$ and let $V$ be a vector field on a neighbourhood of $J$. Take any smooth function $s p(\omega) \rightarrow s p(\omega)$ that coincides with $\mathrm{V}$ on a neighbourhood of $J$ and denote this function again by $V$. It follows from the Koszul formula that the Levi-Civita connection $D$ of the Riemannian manifold $(\mathcal{Z}, G)$ is given by

$$
\left(D_{U} V\right)_{J}=\operatorname{pr}_{J}\left(V^{\prime}(J)(U)\right)
$$

where $V^{\prime}(J) \in \operatorname{End}(s p(\omega))$ is the derivative of the function $V: s p(\omega) \rightarrow s p(\omega)$. Then

$$
\left(D_{U} \mathcal{J} V\right)_{J}=\operatorname{pr}_{J}\left(U V_{J}+J\left(V^{\prime}(J)\right)(U)\right)=J_{p r}\left(V^{\prime}(J)(U)\right)=\mathcal{J}\left(D_{U} V\right)_{J}
$$

Therefore $(G, \mathcal{J})$ is a Kähler structure on $\mathcal{Z}$.

The Lie group $S p(\omega)$ of linear transformations $Q$ of $\mathcal{D}$ that preserve $\omega, \omega(Q x, Q y)$ $=\omega(x, y)$, acts transitively on $\mathcal{Z}$ by conjugation. Indeed, if $J, J^{\prime} \in \mathcal{Z}$, take symplectic bases $E_{\alpha}$ and $E_{\alpha}^{\prime}, \alpha=1, \ldots, 2 n$, determined by $J$ and $J^{\prime}$, respectively. Then, if $Q$ is the linear transformation of $\mathcal{D}$ defined by $Q E_{\alpha}=E_{\alpha}^{\prime}$, we have $Q \in S p(\omega)$ and $J^{\prime}=Q J Q^{-1}$. Denote the isotropy subgroup of $S p(\omega)$ at a point $J^{0} \in \mathcal{Z}$ by $U\left(J^{0}\right)$. Thus $\mathcal{Z}$ is the homogeneous space $S p(\omega) / U\left(J^{0}\right)$. Note also that the complex structure $\mathcal{J}$ and the metric $G$ on $\mathcal{Z}$ are invariant under the action of $S p(\omega)$.

The Lie algebras of the groups $S p(\omega)$ and $U\left(J_{0}\right)$ are $\operatorname{sp}(\omega)$ and $\mathfrak{u}=\{S \in$ $\left.s p(\omega): S J_{0}-J_{0} S=0\right\}$. Set $\mathfrak{m}=\left\{S \in s p(\omega): S J_{0}+J_{0} S=0\right\}$. Then

$$
s p(\omega)=\mathfrak{u} \oplus \mathfrak{m}, \quad[\mathfrak{u}, \mathfrak{m}] \subset \mathfrak{m}, \quad[\mathfrak{m}, \mathfrak{m}] \subset \mathfrak{u} .
$$

Fix a symplectic basis $E_{\alpha}^{0}, \alpha=1, \ldots, 2 n$, such that $J^{0} E_{i}^{0}=E_{i+n}^{0}, J^{0} E_{i+n}^{0}=-E_{i}^{0}$, $i=1, \ldots, n$. Consider the isomorphism of $\mathcal{D}$ with $\mathbb{R}^{2 n}$ that sends $E_{\alpha}^{0}$ to the standard basis of $\mathbb{R}^{2 n}$. Then $S p(\omega) \cong S p(2 n, \mathbb{R})$ and $U\left(J^{0}\right) \cong U(n)$. Thus $\mathcal{Z} \cong$ $S p(\omega) / U\left(J^{0}\right) \cong S p(2 n, \mathbb{R}) / U(n)$ is a symmetric space. Recall that $S p(2 n, \mathbb{R}) / U(n)$ is diffeomorphic to the Siegel upper half plane $\mathbb{S}_{n}$, which is the set of complex symmetric $n \times n$-matrices $Z=X+i Y$ with positive definite imaginary part $Y$ (see, for example, [5] or [9]). Indeed, let

$$
\psi=\left(\begin{array}{cc}
A & B \\
C & D
\end{array}\right)
$$

be the matrix representation of a transformation $\psi \in S p(2 n, \mathbb{R})$ with respect to the standard basis of $\mathbb{R}^{2 n}$. Then

$$
\psi \cdot Z=(A Z+B)(C Z+D)^{-1}
$$


defines a transitive action of $S p(2 n, \mathbb{R})$ on $\mathbb{S}_{n}$. The isotropy subgroup at the matrix $i I \in \mathbb{S}_{n}, I$ being the identity $n \times n$-matrix, is $U(n)$. Thus $S p(2 n, \mathbb{R}) / U(n) \cong \mathbb{S}_{n}$. Let $J(Z)$ be the complex structure corresponding to $Z=X+i Y \in \mathbb{S}_{n}$ under the composite diffeomorphism $\mathbb{S}_{n} \cong S p(2 n, \mathbb{R}) / U(n) \cong \mathcal{Z}$. Denote by $Y^{1 / 2}$ the principal square root of the symmetric positive definite matrix $Y$. Then the matrix

$$
\psi=\left(\begin{array}{cc}
Y^{1 / 2} & X Y^{-1 / 2} \\
0 & Y^{-1 / 2}
\end{array}\right)
$$

represents a transformation in $S p(2 n, \mathbb{R})$ (smoothly depending on $(X, Y))$ such that $\psi \cdot(i I)=Z$. Hence

$$
J(Z)=\psi\left(\begin{array}{cc}
0 & I \\
-I & 0
\end{array}\right) \psi^{-1}=\left(\begin{array}{cc}
-X Y & Y+X Y^{-1} X \\
-Y^{-1} & Y^{-1} X
\end{array}\right) .
$$

It is easy to check by means of the latter formula that the diffeomeorphism $Z \rightarrow$ $J(Z)$ is holomorphic, $\mathbb{S}_{n}$ being considered with its natural complex structure as an open subset of the vector space of symmetric complex matrices. The manifold $\mathbb{S}_{n}$ admits a $S p(2 n, \mathbb{R})$-invariant Kähler metric $H$ introduced and studied by Siegel (see, for example, [7]). For $W=U+i V \in T_{Z} \mathbb{S}_{n}$, it is defined by

$$
H(W, W)=\operatorname{Trace}\left(Y^{-1} U Y^{-1} U+Y^{-1} V Y^{-1} V\right)
$$

One can easily check that the biholomorphism $Z \rightarrow J(Z)$ sends the metric $G$ on $\mathcal{Z}$ to the metric $2 H$ on $\mathbb{S}_{n}$.

2.2. $S p(\omega)$-decomposition of curvature tensors. Let $(M, \omega)$ be a symplectic manifold and $\nabla$ a linear torsion-free connection on $T M$ for which $\nabla \omega=0$. Such a connection always exists [4] (see also [8]). Let $R$ be the curvature tensor of $\nabla$.

Convention. We adopt the following definition for the curvature tensor $R(X, Y)=$ $\nabla_{[X, Y]}-\left[\nabla_{X}, \nabla_{Y}\right]$. This differs by a sign from the definition used in [8, 10].

Set

$$
R(X, Y, Z, U)=\omega(R(X, Y) Z, U), \quad X, Y, Z, U \in T M .
$$

It has been observed in $[8$ that this covariant 4 -tensor has the following properties:

(i) $\quad R(X, Y, Z, U)=-R(Y, X, Z, U)$;

(ii) $\quad R(X, Y, Z, U)=R(X, Y, U, Z)$;

(iii) $\quad R(X, Y, Z, U)+R(Y, Z, X, U)+R(Z, X, Y, U)=0$.

Now consider the space $\mathcal{S}$ of covariant 4 -tensors on a symplectic vector space $(\mathcal{D}, \omega)$ having these properties. The group $S p(\omega)$ acts on the space $\mathcal{S}$ in a natural way. The irreducible decomposition of $\mathcal{S}$ under the action of $S p(\omega)$ has been found in [8]. To describe this decomposition we introduce the Ricci tensor of a tensor $R \in \mathcal{S}$ in the usual way. Let $R(X, Y) Z$ be the $(1,3)$-tensor defined by

$$
\omega(R(X, Y) Z, U)=R(X, Y, Z, U) .
$$

Then the Ricci tensor of $R$ is defined as

$$
\sigma_{R}(X, Y)=\operatorname{Trace}\{Z \rightarrow R(X, Z) Y\} .
$$

Thus, if $E_{1}, \ldots, E_{2 n}$ is a symplectic basis,

$$
\sigma_{R}(X, Y)=\sum_{i=1}^{n}\left[R\left(X, E_{i}, Y, E_{i+n}\right)-R\left(X, E_{i+n}, Y, E_{i}\right)\right] .
$$


It has been shown in 8 that the Ricci tensor $\sigma_{R}$ is symmetric. Let $\mathcal{S}^{0}$ be the subspace of tensors in $\mathcal{S}$ with vanishing Ricci tensor. Denote by $\mathcal{S}^{r}$ the subspace of $\mathcal{S}$ consisting of all tensors in $\mathcal{S}$ of the form

$$
\begin{aligned}
& R(X, Y, Z, U)=\frac{1}{2 n+2}[-\omega(X, Z) P(Y, U)+\omega(Y, U) P(X, Z) \\
& -\omega(X, U) P(Y, Z)+\omega(Y, Z) P(X, U) \\
& -2 \omega(X, Y) P(Z, U)]
\end{aligned}
$$

where $P$ is a symmetric covariant 2 -tensor.

Proposition 1. ([8]) (i) If $n=1$, then $\mathcal{S}$ is an irreducible $S p(\omega)$-module and $\mathcal{S}=\mathcal{S}^{r}$

(ii) If $n>1$, we have the following $S p(\omega)$-irreducible decomposition

$$
\mathcal{S}=\mathcal{S}^{0} \oplus \mathcal{S}^{r} .
$$

The projection to $\mathcal{S}^{r}$ of a tensor $R \in \mathcal{S}$ is given by the right-hand side of (4) with $P=\sigma_{R}([\underline{8})$.

Definition. As in [10], we shall say that a covariant 4-tensor $R$ in $\mathcal{S}$ is of Ricci type if $R \in \mathcal{S}^{r}$ (i.e. if $R$ is a reducible symplectic curvature tensor in the terminology of [8]).

2.3. Contact connections. Let $M$ be a $(2 n+1)$-dimensional contact manifold with contact form $\alpha$. We denote the contact distribution $\operatorname{Ker} \alpha$ by $\mathcal{D}$ and the Reeb vector field by $\xi$.

Following [10, a linear connection $\nabla$ on $T M$ will be called contact, if for every $X \in T M$ and every three sections $Y, Y_{1}, Y_{2}$ of $\mathcal{D}$ and every two sections $X_{1}, X_{2}$ of $T M$

$$
\begin{gathered}
\nabla_{X} Y \text { is a section of } \mathcal{D} \\
\nabla_{\xi} Y=[\xi, Y] \\
\nabla_{X} \xi=0 \\
\left(\nabla_{Y} d \alpha\right)\left(Y_{1}, Y_{2}\right)=0 \\
{\left[X_{1}, X_{2}\right]=\nabla_{X_{1}} X_{2}-\nabla_{X_{2}} X_{1}-d \alpha\left(X_{1}, X_{2}\right) \xi .}
\end{gathered}
$$

The last identity implies $\nabla_{Y_{1}} Y_{2}-\nabla_{Y_{2}} Y_{1}=\left[Y_{1}, Y_{2}\right]-\alpha\left(\left[Y_{1}, Y_{2}\right]\right) \xi=$ the projection of $\left[Y_{1}, Y_{2}\right]$ to $\mathcal{D}$ with respect to the decomposition $T M=\mathcal{D} \oplus \mathbb{R} \xi$. Note also that $[\xi, Y]=\nabla_{\xi} Y \in \mathcal{D}$, so $\alpha([\xi, Y])=0$.

Proposition 2. ([10]) Every contact manifold admits a contact connection. The set of all contact connections on $(M, \alpha)$ is an affine space modeled on the space of symmetric covariant 3 -tensors on the contact distribution $\mathcal{D}$.

Henceforward $\nabla$ will denote a contact connection on $M$.

It is convenient to set $\omega=d \alpha$. Then

$$
\omega(\xi, .)=0,
$$

while $\omega$ is a symplectic form on $\mathcal{D}$. 
Lemma 1. For every contact connection $\nabla$

$$
\nabla \omega=0 .
$$

Proof. Let $X, Y, Z$ be vector fields on $M$. If $X, Y, Z$ are sections of $\mathcal{D}$, then $\left(\nabla_{X} \omega\right)(Y, Z)=0$ by (8). Moreover, in view of (6),

$$
\begin{gathered}
\left(\nabla_{\xi} \omega\right)(Y, Z)=\xi(d \alpha(Y, Z))-d \alpha\left(\nabla_{\xi} Y, Z\right)-d \alpha\left(Y, \nabla_{\xi} Z\right) \\
=\xi(d \alpha(Y, Z))-d \alpha([\xi, Y], Z)-d \alpha(Y,[\xi, Z])=d(d \alpha)(\xi, Y, Z)=0 .
\end{gathered}
$$

We also have $\left(\nabla_{X} \omega\right)(\xi, Z)=0$ for every $X, Z \in T M$ since $\omega(\xi,)=$.0 and $\nabla_{X} \xi=$ 0 .

Lemma 2. The curvature tensor $R$ of a contact connection satisfies the following identities

(i) $\quad \omega(R(X, Y) Z, U)=\omega(R(X, Y) U, Z), \quad X, Y, Z, U \in T M$;

(ii) $\quad R(X, Y) Z+R(Y, Z) X+R(Z, Y) X=0$ (the Bianchi identity).

Proof. Since $\nabla \omega=0$, we have

$$
\begin{gathered}
\omega\left(\nabla_{X} \nabla_{Y} Z, U\right)=X Y(\omega(Z, U))-X\left(\omega\left(Z, \nabla_{Y} U\right)\right)-Y\left(\omega\left(Z, \nabla_{X} U\right)\right) \\
+\omega\left(Z, \nabla_{Y} \nabla_{X} U\right) \\
\quad \text { and } \\
\omega\left(\nabla_{[X, Y]} Z, U\right)=[X, Y](\omega(Z, U))-\omega\left(Z, \nabla_{[X, Y]} U\right) .
\end{gathered}
$$

It follows that $\omega(R(X, Y) Z, U)=-\omega(Z, R(X, Y) U)$. This proves $(i)$.

To prove (ii) we first note that $R(\cdot, \cdot) \xi=0$ since $\nabla \xi=0$, and that if $Z \in \mathcal{D}$, then $R(\cdot, \cdot) Z \in \mathcal{D}$ since $\nabla$ preserves the bundle $\mathcal{D}$. Thus, to show the Binachi identity, it is enough to consider the cases when $X, Y, Z \in \mathcal{D}$ and $X, Z \in \mathcal{D}, Y=\xi$. In the first case the Bianchi identity has been proved in [10, Lemma 2.6]. In the second case, we have

$$
\begin{gathered}
R(X, \xi) Z+R(\xi, Z) X=-\nabla_{X} \nabla_{\xi} Z+\nabla_{\xi}\left(\nabla_{X} Z-\nabla_{Z} X\right)+\nabla_{Z} \nabla_{\xi} X \\
+\nabla_{[X, \xi]} Z+\nabla_{[\xi, Z]} X \\
=-\nabla_{X}[\xi, Z]+\nabla_{\xi}[X, Z]_{\mathcal{D}}-\nabla_{Z}[X, \xi]+\nabla_{[X, \xi]} Z+\nabla_{[\xi, Z]} X-\nabla_{[X, Z]} \xi \\
=(-[X,[\xi, Z]]+[\xi,[X, Z]]-[Z,[X, \xi]])_{\mathcal{D}}-\xi(\alpha([X, Z])) \xi \\
=-\xi(\alpha([X, Z])) \xi .
\end{gathered}
$$

where the subscript $\mathcal{D}$ means "the projection to $\mathcal{D}$ ". It follows that $R(X, \xi) Z+$ $R(\xi, Z) X=0$ since the left-hand side of the identity above lies in $\mathcal{D}$.

\section{Almost contact metric structures on contact twistor spaces}

Let $M$ be a contact manifold with contact form $\alpha$, contact distribution $\mathcal{D}$ and Reeb field $\xi, \operatorname{dim} M=2 n+1$. Set $\omega=d \alpha$ as above.

Following [10] we define the contact twistor space of $(M, \alpha)$ as the bundle $\mathcal{C} \rightarrow M$ whose fibre at every point $p \in M$ is $\mathcal{Z}\left(\mathcal{D}_{p}, \omega_{p}\right)$, the space of complex structures on the vector space $\mathcal{D}_{p}$ compatible with the symplectic form $\omega_{p}\left|\mathcal{D}_{p}=d \alpha\right| \mathcal{D}_{p}$.

The total space $\mathcal{C}$ is a submanifold of $\operatorname{End}(\mathcal{D})$. We imbed $\operatorname{End}(\mathcal{D})$ into $\operatorname{End}(T M)$ setting $A \xi=0$ for every $A \in \operatorname{End}(\mathcal{D})$, and shall consider $\mathcal{C} \rightarrow M$ as a subbundle of the bundle $\pi: \operatorname{End}(T M) \rightarrow M$. 
Remark 1. According to this convention, every $J \in \mathcal{C}$ will be considered as an endomorphism of $T_{\pi(J)} M$ such that

$$
\begin{gathered}
J^{2} X=-X+\alpha(X) \xi, \quad X \in T_{\pi(J)} M, \\
\omega(J X, J Y)=\omega(X, Y), \quad X, Y \in T_{\pi(J)} M, \\
\omega(Z, J Z)>0 \text { for } Z \in \mathcal{D}_{\pi(J)}, Z \neq 0 .
\end{gathered}
$$

Suppose we a given a contact connection $\nabla$ on $T M$.

The connection $\nabla$ induces a connection on the vector bundle $\operatorname{End}(T M)$ which will also be denoted by $\nabla$.

Remark 2. Let $S$ be a section of the bundle $\mathcal{C} \rightarrow M$. Denote the extension of $S$ to a section of $\operatorname{End}(T M)$ by $\bar{S}$, so $\bar{S}_{p} \mid \mathcal{D}_{p}=S_{p}, \bar{S}_{p}\left(\xi_{p}\right)=0, p \in M$. Then $\left(\nabla_{X} \bar{S}\right)(Z)=\left(\nabla_{X} S\right)(Z)$ for $X \in T_{p} M, Z \in \mathcal{D}_{p}$ since $\nabla$ preserves the bundle $\mathcal{D}$. Also $\left(\nabla_{X} \bar{S}\right)(\xi)=0$ since $\nabla \xi=0$. Thus, the extension of $\nabla_{X} S$ is $\nabla_{X} \bar{S}$.

Let $\mathcal{H}$ be the horizontal subbundle of $T E n d(T M)$ defined by means of the connection $\nabla$ on $\operatorname{End}(T M)$.

Notation. Let $J \in \mathcal{C}$ and $p=\pi(J)$. Take a basis $e_{1}, \ldots, e_{n}, e_{n+1}=J e_{1}, \ldots, e_{2 n}=$ $J e_{n}$ of $\mathcal{D}_{p}=\operatorname{Im} J$ that is orthonormal with respect to the metric $g_{J}(u, v)=\omega(u, J v)$ on $D_{p}$. For this basis $\omega\left(e_{i}, e_{j}\right)=\omega\left(e_{i+n}, e_{j+n}\right)=0, \omega\left(e_{i}, e_{j+n}\right)=\delta_{i j}, i, j=$ $1, \ldots, n$. Since by Lemma $11 \omega$ is $\nabla$-parallel, there exists a frame of vector fields $E_{1}, \ldots, E_{2 n}$ in a (geodesically convex) neighbourhood of $p$ such that

$$
\begin{gathered}
E_{r}(p)=e_{r},\left.\quad \nabla E_{r}\right|_{p}=0, \quad r=1, \ldots, 2 n \\
\omega\left(E_{i}, E_{j}\right)=\omega\left(E_{i+n}, E_{j+n}\right)=0, \quad \omega\left(E_{i}, E_{j+n}\right)=\delta_{i j}, \quad i, j=1, \ldots, n .
\end{gathered}
$$

Define a section $S$ of $\operatorname{End}(T M)$ by

$$
S E_{i}=E_{i+n}, \quad S E_{i+n}=-E_{i}, \quad i=1, \ldots, n, \quad S \xi=0 .
$$

Then $S$ is a section of $\mathcal{C}$ such that

$$
S(p)=J,\left.\quad \nabla S\right|_{p}=0 .
$$

It follows that, for every $J \in \mathcal{C}$ and $X \in T_{\pi(J)} M$, the horizontal lift $X_{J}^{h}=$ $S_{*}(X) \in \mathcal{H}_{J}$ of $X$ lies in $T_{J} \mathcal{C}$, i.e. the horizontal spaces $\mathcal{H}_{J}, J \in \mathcal{C}$, are tangent to the manifold $\mathcal{C}$. Thus, if $\mathcal{V}_{J}=\operatorname{Ker}(\pi \mid \mathcal{C})_{*}$ is the vertical space of the bundle $\mathcal{C} \rightarrow M$, we have the direct sum decomposition

$$
T_{J} \mathcal{C}=\mathcal{V}_{J} \oplus \mathcal{H}_{J}
$$

Let $\left(U, x_{1}, \ldots, x_{2 n+1}\right)$ be a local coordinate system of $M$. Define a frame $L_{\alpha \beta}$ of $\operatorname{End}(T M)$ setting $L_{\alpha \beta} E_{\gamma}=\delta_{\alpha \gamma} E_{\beta}, 1 \leq \alpha, \beta, \gamma \leq 2 n+1$. If $L \in \pi^{-1}(U) \subset$ $\operatorname{End}(T M)$, we have

$$
L=\sum_{\beta, \gamma=1}^{2 n+1} y^{\beta \gamma} L_{\beta \gamma}
$$

for some smooth functions $y^{\beta \gamma}$. Set $\widetilde{x}^{\alpha}(L)=x_{\alpha} \circ \pi(L)$. Then $\left(\widetilde{x}^{\alpha}, y^{\beta \gamma}\right)$ is a local coordinate system of the manifold $\operatorname{End}(T M)$. 
Let $\left[\theta_{\alpha \beta}^{\mu \nu}\right]$ be the connection matrix of $\nabla$ with respect to the frame $L_{\alpha \beta}$ :

$$
\nabla_{X} L_{\alpha \beta}=\sum_{\mu, \nu=1}^{2 n+1} \theta_{\alpha \beta}^{\mu \nu}(X) L_{\mu \nu}, \quad X \in T M .
$$

Then, for every vector field

$$
X=\sum_{\alpha=1}^{2 n+1} X^{\alpha} \frac{\partial}{\partial x_{\alpha}}
$$

on $U$, the horizontal lift $X^{h}$ on $\pi^{-1}(U)$ is given by

$$
X^{h}=\sum_{\alpha=1}^{2 n+1}\left(X^{\alpha} \circ \pi\right) \frac{\partial}{\partial \widetilde{x}^{\alpha}}-\sum_{\beta, \gamma, \mu, \nu=1}^{2 n+1} y^{\beta \gamma}\left(\theta_{\beta \gamma}^{\mu \nu}(X) \circ \pi\right) \frac{\partial}{\partial y^{\mu \nu}} .
$$

Let $L \in \operatorname{End}(T M)$ and $p=\pi(L)$. Then (14) implies that under the standard identification $T_{L} \operatorname{End}\left(T_{p} M\right) \cong \operatorname{End}\left(T_{p} M\right)$ we have

$$
\left[X^{h}, Y^{h}\right]_{L}=[X, Y]_{L}^{h}+R(X, Y) L,
$$

where $R(X, Y) L$ is the curvature of the connection $\nabla$ on $\operatorname{End}(T M)$.

Remark 3. Note that, for $J \in \mathcal{C}$, the isomorphism $T_{J} \operatorname{End}\left(T_{\pi(J)} M\right) \cong \operatorname{End}\left(T_{\pi(J)} M\right)$ identifies the vertical space $\mathcal{V}_{J}$ of the bundle $\mathcal{C} \rightarrow M$ with the space of endomorphisms $U$ of $T_{\pi(J)} M$ such that $U \xi=0, J U+U J=0, \omega(U X, Y)+\omega(X, U Y)=0$, $X, Y \in T_{\pi(J)} M$.

Remark 4. Given $J \in \mathcal{C}$, denote for a moment the extension of the endomorphism $J$ of $\mathcal{D}_{p}, p=\pi(J)$, to an endomorphism of $T_{p} M$ by $\bar{J}\left(\bar{J} \mid \mathcal{D}_{p}=J, \bar{J} \xi=0\right)$. Then, for $X, Y \in T_{p} M, R(X, Y) \bar{J}$ is the extension of the endomorphism $R(X, Y) J$ of $\mathcal{D}_{p}$ since $\nabla$ preserves $\mathcal{D}$ and $\nabla \xi=0$.

Remarks 1-4 show that the imbedding $\operatorname{End}(\mathcal{D}) \hookrightarrow \operatorname{End}(T M)$ has nice properties in the context of our considerations.

As usual in twistor theory, we can define two endomorphisms $\Phi_{k}$ of $T \mathcal{C}$ setting

$$
\Phi_{k} X_{J}^{h}=(J X)_{J}^{h} \text { for } X \in T_{\pi(J)} M, \quad \Phi_{k} V=(-1)^{k+1} J V \text { for } V \in \mathcal{V}_{J} .
$$

Clearly, $\Phi_{k}^{3}+\Phi_{k}=0, \operatorname{rank} \Phi_{k}=2 n$. Recall that an endomorphism of the tangent bundle of a manifold with these properties is called a partially complex structure or a $f$-structure. Note also that $\Phi_{k}\left(\xi^{h}\right)=0$.

The fibre of the subbundle $\operatorname{Im} \Phi_{k}$ of $T \mathcal{C}$ at a point $J \in \mathcal{C}$ is the space $\mathcal{V}_{J} \oplus$ $\left\{X_{J}^{h}: X \in \mathcal{D}_{\pi(J)}\right\}$. Set $\mathcal{E}=\operatorname{Im} \Phi_{1}\left(=\operatorname{Im} \Phi_{2}\right)$. Then $\left(\mathcal{E}, \Phi_{k} \mid \mathcal{E}\right)$ is an almost $C R$ structure on $\mathcal{C}$. For $k=1$, the integrability condition for this structure has been obtained in [10].

For every $t>0$, we define a Riemannian metric $G_{t}$ on $\mathcal{C}$ as follows: Let $J \in \mathcal{C}$ and $p=\pi(J)$. For $X, Y \in \mathcal{D}_{p}$, we set $G_{t}\left(X_{J}^{h}, Y_{J}^{h}\right)=\omega(X, J Y)$ and $G_{t}\left(X_{J}^{h}, \xi_{J}^{h}\right)=0$. Thus

$$
G_{t}\left(X_{J}^{h}, Y_{J}^{h}\right)=\omega(X, J Y)+\alpha(X) \alpha(Y) \text { for every } X, Y \in T_{p} M .
$$

On the vertical subspace $\mathcal{V}_{J}$ of $T_{J} \mathcal{C}$, we set $G \mid \mathcal{V}_{J}=t G_{J}, t$-times the metric on the fibre through $J$. Finally, the horizontal and vertical spaces at $J$ are declared to be orthogonal. Then $\left(\Phi_{k}, \xi^{h}, G_{t}\right)$ is an almost contact metric structure on $\mathcal{C}$. We refer to 1 for general facts about (almost) contact metric structures.

The main purpose of this section is to find conditions on $M$ under which $\left(\Phi_{k}, \xi^{h}, G_{t}\right)$ is a normal structure. Recall that any almost contact metric structure $(\varphi, \xi, g)$ on 
a manifold $N$ induces an almost complex structure $K$ on the manifold $N \times S^{1}$ for which $K X=\varphi X$ for $X \in T N, X \perp \xi, K \xi=-\frac{\partial}{\partial s} \in T S^{1}$ where $s$ is the local coordinate $e^{2 \pi i s} \rightarrow s$ on $S^{1}$. The structure $(\varphi, \xi, g)$ is said to be normal if the induced almost complex structure on $N \times S^{1}$ is integrable. It is well-known that $(\varphi, \xi, g)$ is a normal structure if and only if the tensor $N^{(1)}(X, Y)=\varphi^{2}[X, Y]+[\varphi X, \varphi Y]-$ $\varphi[\varphi X, Y]-\varphi[X, \varphi Y]+d \eta(X, Y) \xi$ vanishes, where $\eta(X)=g(X, \xi)$ (see, for example, [1] the definition of $d \eta$ used here is twice the one in [1). For the almost contact structure $\left(\Phi_{k}, \xi^{h}, G_{t}\right)$ this tensor will be denoted by $N_{k}^{(1)}$.

Let $A$ be a (local) section of $\operatorname{End}(T M)$ with $A \xi=0$ (i.e. a section of $\operatorname{End}(\mathcal{D})$ ). Define a section $A^{*}$ of $\operatorname{End}(\mathcal{D})$ by $\omega\left(A^{*} X, Y\right)=\omega(X, A Y), X, Y \in T M$, and consider it as a section of $\operatorname{End}(T M)\left(A^{*} \xi=0\right)$. Then

$$
\check{A}=\frac{1}{2}\left(A-A^{*}\right)
$$

is an $\omega$-skew-symmetric section of $\operatorname{End}(T M)$, and we can define a vertical vector field $\widetilde{A}$ on $\mathcal{C}$ setting

$$
\widetilde{A}_{J}=\frac{1}{2}\left(\check{A}_{\pi(J)}+J \circ \check{A}_{\pi(J)} \circ J\right) .
$$

Lemma 3. If $J \in \mathcal{C}$ and $X$ is a vector field near the point $p=\pi(J)$, then

(i) $\left[X^{h}, \widetilde{A}\right]_{J}=\left(\widetilde{\nabla_{X} A}\right)_{J}$,

(ii) $\left[X^{h}, \Phi_{k} \widetilde{A}\right]_{J}=\Phi_{k}\left(\widetilde{\nabla_{X} A}\right)_{J}, \quad k=1,2$,

(iii) $\left[\Phi_{k} X^{h}, \widetilde{A}\right]_{J}=\left(\widetilde{\nabla_{J X} A}\right)_{J}-\left(\widetilde{A}_{J} X\right)_{J}^{h}$,

(iv) $\left[\Phi_{k} X^{h}, \Phi_{k} \widetilde{A}\right]_{J}=\Phi_{k}\left(\widetilde{\nabla_{J X} A}\right)_{J}-\left(\Phi_{k}\left(\widetilde{A}_{J}\right) X\right)_{J}^{h}$.

Proof. Note first that by (14)

$$
\left[X^{h}, \frac{\partial}{\partial y^{\beta \gamma}}\right]_{J}=0, \quad X_{J}^{h}=\sum_{\alpha=1}^{2 n+1} X^{\alpha}(p)\left(\frac{\partial}{\partial \widetilde{x}^{\alpha}}\right)_{J} .
$$

Let $A E_{\alpha}=\sum_{\beta=1}^{2 n+1} a^{\alpha \beta} E_{\beta}, A^{*} E_{\alpha}=\sum_{\beta=1}^{2 n+1} a^{* \alpha \beta} E_{\beta}, \check{A} E_{\alpha}=\sum_{\beta=1}^{2 n+1} \check{a}^{\alpha \beta} E_{\beta}$. Then

$$
\widetilde{A}=\sum_{\alpha, \beta=1}^{2 n+1} \widetilde{a}^{\alpha \beta} \frac{\partial}{\partial y^{\alpha \beta}},
$$

where

$$
\widetilde{a}^{\alpha \beta}=\frac{1}{2}\left[\check{a}^{\alpha \beta} \circ \pi+\sum_{\mu, \nu=1}^{2 n+1} y^{\alpha \mu}\left(\check{a}^{\mu \nu} \circ \pi\right) y^{\nu \beta}\right], \quad \check{a}^{\alpha \beta}=\frac{1}{2}\left(a^{\alpha \beta}-a^{* \alpha \beta}\right) .
$$

In view of (17), it follows that

$$
\left[X^{h}, \widetilde{A}\right]_{J}=\frac{1}{2} \sum_{\alpha, \beta=1}^{2 n+1}\left\{X_{p}\left(\check{a}^{\alpha \beta}\right)+\sum_{\mu, \nu=1}^{2 n+1} y^{\alpha \mu}(J) X_{p}\left(\check{a}^{\mu \nu}\right) y^{\nu \beta}(J)\right\}\left(\frac{\partial}{\partial y^{\alpha \beta}}\right)_{J} .
$$


On the other hand, we have $\left(\nabla_{X_{p}} \check{A}\right)\left(E_{\alpha}\right)=\sum_{\beta=1}^{2 n+1} X_{p}\left(\check{a}^{\alpha \beta}\right)\left(E_{\beta}\right)_{p}$. Note also that

$$
\omega\left(\left(\nabla_{X} A^{*}\right)(Y), Z\right)=\omega\left(Y,\left(\nabla_{X} A\right)(Z)\right)
$$

for every $Y, Z \in \mathcal{D}$ where $\left(\nabla_{X} A^{*}\right)(Y) \in \mathcal{D}_{p}$. Moreover, $\left(\nabla_{X} A^{*}\right)(\xi)=0$. Thus $\left(\nabla_{X} A\right)^{*}=\nabla_{X} A^{*}$, hence $\left(\nabla_{X} A\right)^{r}=\nabla_{X} \check{A}$. It follows that

$$
\left(\widetilde{\nabla_{X} A}\right)_{J}=\frac{1}{2}\left(\nabla_{X_{p}} \check{A}-J \circ \nabla_{X_{p}} \check{A} \circ J\right) .
$$

Therefore the right-hand side of $(20)$ equals $\left(\widetilde{\nabla_{X} A}\right)_{J}$.

The second formula of the lemma can be proved by similar computations taking into account that

$$
\Phi_{k} \widetilde{A}=(-1)^{k+1} \sum_{\alpha, \beta, \gamma=1}^{2 n+1} y^{\alpha \beta} \widetilde{a}^{\beta \gamma} \frac{\partial}{\partial y^{\alpha \gamma}} .
$$

Set $X=\sum_{\alpha=1}^{2 n+1} f^{\alpha} E_{\alpha}$. Then

$$
\Phi_{k} X^{h}=\sum_{\alpha, \beta=1}^{2 n+1}\left(f^{\alpha} \circ \pi\right) y^{\alpha \beta} E_{\beta}^{h} .
$$

This and the first formula of the lemma imply (iii).

Formula (iv) follows from (ii) and (22).

Lemma 4. For every two vector fields $X, Y$ near the point $p=\pi(J)$ and every two integers $a, b \geq 0$, we have

$$
\left[\Phi_{k}^{a} X^{h}, \Phi_{k}^{b} Y^{h}\right]_{J}=\left[S^{a} X, S^{b} Y\right]_{J}^{h}+R_{p}\left(J^{a} X, J^{b} Y\right) J, \quad k=1,2 .
$$

Proof. This follows from the identities $\left(\Phi_{k}^{a} X\right)_{J}^{h}=S_{* p}\left(J^{a} X_{p}\right), \Phi_{k}^{a} X^{h} \circ S=\left(S^{a} X\right)^{h} \circ$ $S$ and formula (15).

Denote by $D=D_{t}$ the Levi-Civita connection of the metric $G_{t}$.

Lemma 5. If $X, Y, Z$ are vector fields on a neighbourhood of the point $p=\pi(J)$, then

$$
\begin{gathered}
G_{t}\left(D_{X^{h}} Y^{h}, Z^{h}\right)_{J}=G_{t}\left(\left(\nabla_{X} Y\right)^{h}, Z^{h}\right)_{J}+\left[X_{p}(\alpha(Y))-\alpha_{p}\left(\nabla_{X} Y\right)\right] \alpha_{p}(Z) \\
+\frac{1}{2}\left[\alpha_{p}(X) \omega_{p}(Y, Z)+\alpha_{p}(Y) \omega_{p}(X, Z)-\alpha_{p}(Z) \omega_{p}(X, Y)\right]
\end{gathered}
$$

Proof. Take a local section $S$ of $\mathcal{C}$ such that $S(p)=J$ and $\left.\nabla S\right|_{p}=0$. Then by the Koszul formula and (15) we have

$$
\begin{gathered}
2 G_{t}\left(D_{X^{h}} Y^{h}, Z^{h}\right)_{J}=X_{p}(\omega(Y, S Z))+X(\alpha(Y) \alpha(Z)) \\
+Y_{p}(\omega(Z, S X))+Y(\alpha(Z) \alpha(X))-Z_{p}(\omega(X, S Y))-Z(\alpha(X) \alpha(Y)) \\
+\omega_{p}(Z, J[X, Y))+\alpha_{p}(Z) \alpha_{p}([X, Y])+\omega_{p}(Y, J[Z, X])+\alpha_{p}(Y) \alpha_{p}([Z, X]) \\
+\omega_{p}(X, J[Z, Y])+\alpha_{p}(X) \alpha_{p}([Z, Y]) .
\end{gathered}
$$

It follows from Lemma 1 that

$$
X_{p}(\omega(Y, S Z))=\omega\left(\nabla_{X_{p}} Y, J Z\right)+\omega\left(Y, J \nabla_{X_{p}} Z\right)
$$

in view of the identity $\left.\nabla S\right|_{p}=0$. Moreover, we have

$$
\omega(Z, J[X, Y])=\omega\left(Z, J \nabla_{X} Y\right)-\omega\left(Z, J \nabla_{Y} X\right)
$$


by (9) and (10). Also,

$$
\alpha(Z) \alpha([X, Y])=-\alpha(Z) \omega(X, Y)+[X(\alpha(Y))-Y(\alpha(X))] \alpha(Z)
$$

since $\omega=d \alpha$.

These identities easily imply the lemma.

Notation. Let $A_{1}, \ldots, A_{n^{2}+n}$ be sections of $\operatorname{End}(\mathcal{D})$ such that $A_{1}(p), \ldots, A_{n^{2}+n}(p)$ is a basis of the vertical space $\mathcal{V}_{J} \subset \operatorname{End}\left(T_{p} M\right)$ and $\left.\nabla A_{\varepsilon}\right|_{p}=0, \varepsilon=1, \ldots, n^{2}+n$.

Then the vector fields $\widetilde{A}_{\varepsilon}$ constitute a frame of the vertical bundle in a neighbourhood of $J$.

The Koszul formula and Lemma $3(i)$ imply that $\left(D_{\widetilde{A}_{\varepsilon}} \widetilde{A}_{\delta}\right)_{J}$ is orthogonal to every horizontal vector $X_{J}^{h}, X \in T_{p} M$. Thus we have the following

Lemma 6. The fibres of the bundle $\pi: \mathcal{C} \rightarrow M$ are totally geodesic submanifolds.

Lemma 7. If $X, Y$ are vector fields on a neighbourhood of the point $p=\pi(J)$ and $V$ is a vertical vector field in a neighbourhood of $J$, then

$$
\begin{aligned}
& G_{t}\left(D_{X^{h}} Y^{h}, V\right)_{J}=\frac{1}{2}\left[-\omega\left(X_{p}, V_{J} Y_{p}\right)+G_{t}\left(R_{p}(X, Y) J, V_{J}\right)\right], \\
& D_{V} X^{h}=\mathcal{H} D_{X^{h}} V, \quad G_{t}\left(D_{V} X^{h}, Y^{h}\right)_{J}=-G_{t}\left(D_{X^{h}} Y^{h}, V\right)_{J},
\end{aligned}
$$

where $\mathcal{H}$ means "the horizontal component".

Proof. The Koszul formula, Lemma 3 (i) and identity (15) imply

$$
2 G_{t}\left(D_{X^{h}} Y^{h}, \widetilde{A}_{\varepsilon}\right)_{J}=-\left(\widetilde{A}_{\varepsilon}\right)_{J}\left(G_{t}\left(X^{h}, Y^{h}\right)\right)+G_{t}\left(R_{p}(X, Y) J, \widetilde{A}_{\varepsilon}\right) .
$$

Let $\gamma$ be a curve in the fibre of $\mathcal{C}$ through the point $J$ such that $\gamma(0)=J$ and $\dot{\gamma}(0)=\left(A_{\varepsilon}\right)_{J}$. Then

$$
\begin{aligned}
\left(\widetilde{A}_{\varepsilon}\right)_{J}\left(G_{t}\left(X^{h}, Y^{h}\right)\right) & =\left.\frac{d}{d t}\left(\omega\left(X_{p}, \gamma(t) Y_{p}\right)+\alpha\left(X_{p}\right) \alpha\left(Y_{p}\right)\right)\right|_{t=0} \\
& =\omega\left(X_{p},\left(A_{\varepsilon}\right)_{J} Y_{p}\right) .
\end{aligned}
$$

This proves the first formula of the lemma.

By Lemma 6] $D_{V} X^{h}$ is orthogonal to every vertical vector field, thus it is horizontal. Moreover, $\left[V, X^{h}\right]$ is a vertical vector field, hence $D_{V} X^{h}=\mathcal{H} D_{X^{h}} V$. We also have

$$
G_{t}\left(D_{V} X^{h}, Y^{h}\right)=G_{t}\left(\left[V, X^{h}\right]+D_{X^{h}} V, Y^{h}\right)=-G_{t}\left(V, D_{X^{h}} Y^{h}\right) .
$$

Lemmas 5 and 7 imply the following.

Corollary 1. Let $X, Y \in T_{p} M$ and $V, W \in \mathcal{V}_{J}$. Then

$$
\begin{gathered}
G_{t}\left(D_{X^{h}} \xi^{h}, Y^{h}\right)_{J}=\frac{1}{2} \omega(X, Y), \quad G_{t}\left(D_{V} \xi^{h}, W\right)=0 \\
G_{t}\left(D_{X^{h}} \xi^{h}, V\right)_{J}=G_{t}\left(D_{V} \xi^{h}, X^{h}\right)_{J}=\frac{1}{2} G_{t}\left(R\left(X, \xi_{p}\right) J, V\right) .
\end{gathered}
$$

\section{Corollary 2.}

$$
d \eta_{t}\left(X^{h}, Y^{h}\right)=\omega(X, Y), \quad d \eta_{t}\left(X^{h}, V\right)=d \eta_{t}(V, W)=0, \quad \delta \eta_{t}=0 .
$$

Corollary 3. Every integral curve of $\xi^{h}$ is a geodesic in $\left(\mathcal{C}, G_{t}\right)$. 
Corollary 4. The vector field $\xi^{h}$ on $\left(\mathcal{C}, G_{t}\right)$ is Killing if and only if $R(X, \xi) Y=0$ for every $X, Y \in T M$.

Proof. By Corollary 1, $\xi^{h}$ is Killing if and only if $R(X, \xi) J=0$ for every $J \in \mathcal{C}$ and $X \in T_{\pi(J)} M$. Fix a tangent vector $X \in T_{p} M$ and suppose that $R(X, \xi) J=0$ for every $J$ in the fibre $\mathcal{C}_{p}$ of the bundle $\mathcal{C}$. Let $e_{1}, \ldots, e_{2 n}$ be a symplectic basis of $\mathcal{D}_{p}$ and let $J$ be the complex structure of $\mathcal{D}_{p}$ corresponding to this basis, $J e_{i}=e_{i+n}$, $i=1, \ldots, n$. Then $J \in \mathcal{C}_{p}$ and the identity $R(X, \xi) J=0$ implies

$$
\begin{gathered}
\omega\left(R(X, \xi) e_{i+n}, e_{k}\right)+\omega\left(R(X, \xi) e_{i}, e_{k+n}\right)=0, \quad i, k=1, \ldots, n, \\
\omega\left(R(X, \xi) e_{i+n}, e_{k+n}\right)-\omega\left(R(X, \xi) e_{i}, e_{k}\right)=0 .
\end{gathered}
$$

For $\lambda \in \mathbb{R}$, as in 9, consider the symplectic basis $e_{i}^{\prime}=e_{i}, e_{i+n}^{\prime}=\lambda e_{i}+e_{i+n}$. Applying (25) for this basis, we get $\omega\left(R(X, \xi) e_{i}, e_{k}\right)=0$, hence $\omega\left(R(X, \xi) e_{i+n}, e_{k+n}\right)=0$ by (26). It follows from the identities

$$
\omega\left(R(X, \xi) e_{i}, e_{k}\right)=\omega\left(R(X, \xi) e_{i+n}, e_{k+n}\right)=0,
$$

Lemma 2 (i), and identity (25) that $\omega(R(X, \xi) Z, Z)=0$ for every $Z \in \mathcal{D}_{p}$. In view of Lemma 2 (i), polarization of the latter identity gives $\omega(R(X, \xi) Y, Z)=0$ for $Y, Z \in \mathcal{D}_{p}$. Therefore $R(X, \xi) Y=0$ for $Y \in \mathcal{D}_{p}$. For $Y=\xi$ this is obvious.

Conversely, if $R(X, \xi) Y=0$ for every $X, Y$, we have clearly $R(X, \xi) J=0$, so $\xi^{h}$ is Killing.

Proposition 3. Let $J \in \mathcal{C}, X, Y \in T_{\pi(J)} M, V, W \in \mathcal{V}_{J}$. Then

$$
\begin{gathered}
N_{k}^{(1)}\left(X^{h}, Y^{h}\right)_{J}=-R(X, Y) J+R(J X, J Y) J \\
-(-1)^{k+1} J(R(J X, Y) J+R(X, J Y) J), \\
N_{k}^{(1)}\left(X^{h}, V\right)=\left[1+(-1)^{k}\right](J V X)_{J}^{h}, \quad N_{k}^{(1)}(V, W)=0 .
\end{gathered}
$$

Proof. Extending $X, Y$ to vector fields in a neighbourhood of the point $p=\pi(J)$ and taking into account Lemma 4. Corollary 2, and identity (9), we easily see that

$$
\begin{gathered}
\mathcal{H} N_{k}^{(1)}\left(X^{h}, Y^{h}\right)_{J}= \\
\left(-S_{p}\left(\nabla_{X} S\right)_{p}(Y)+S_{p}\left(\nabla_{Y} S\right)_{p}(X)+\left(\nabla_{S X} S\right)_{p}(Y)-\left(\nabla_{S Y} S\right)_{p}(X)\right)_{J}^{h} \\
-d \alpha\left(S X_{p}, S Y_{p}\right) \xi_{J}^{h}+\omega\left(X_{p}, Y_{p}\right) \xi_{J}^{h},
\end{gathered}
$$

$S$ being defined in Section 3. We have $d \alpha\left(S X_{p}, S Y_{p}\right)=\omega\left(J X_{p}, J Y_{p}\right)=\omega\left(X_{p}, Y_{p}\right)$. Hence

$$
\mathcal{H} N_{k}^{(1)}\left(X^{h}, Y^{h}\right)_{J}=0
$$

since $\left.\nabla S\right|_{p}=0$. Now the first formula of the lemma follows from Lemma 4 and the fact that $\Phi_{k}^{2}=-I d$ on the vertical spaces.

Let $A$ be a section of $\operatorname{End}(\mathcal{D})$ such that $A_{p}=V$. Denote by $\widetilde{A}$ the vertical vector field on $\mathcal{C}$ defined by (16). Then

$$
N_{k}^{(1)}\left(X^{h}, V\right)=N_{k}^{(1)}\left(X^{h}, \widetilde{A}\right)_{J}=\left[1+(-1)^{k}\right]\left(J \widetilde{A}_{J} X\right)_{J}^{h}
$$

by Lemma 3 and Corollary 2 .

Corollary 2 and the fact that $\Phi_{k}$ is a complex structure on the fibres of $\mathcal{C}$ imply $N_{k}^{(1)}(V, W)=0$. 
Now we set

$$
R_{\mathcal{D}}(X, Y, Z, T)=\omega(R(X, Y) Z, T) \text { for } X, Y, Z, T \in \mathcal{D} .
$$

Note that this covariant 4-tensor satisfies the identities $(i),(i i),(i i i)$ in Section 2.2

Theorem 1. (i) The almost contact metric structure $\left(\Phi_{1}, \xi^{h}, G_{t}\right)$ is normal if and only if $R(X, \xi) Y=0$ for every $X, Y \in T M$ and the tensor $R_{\mathcal{D}}$ is of Ricci type.

(ii) The almost contact metric structure $\left(\Phi_{1}, \xi^{h}, G_{t}\right)$ is never normal.

Proof. By Proposition 3. $\left(\Phi_{1}, \xi^{h}, G_{t}\right)$ is a normal structure if and only if $N_{1}^{(1)}\left(X^{h}, Y^{h}\right)_{J}=$ 0 for every $J \in \mathcal{C}$ and $X, Y \in T_{\pi(J)} M$.

Note first that $N_{1}^{(1)}\left(X^{h}, Y^{h}\right)_{J}$, with $J, X, Y$ fixed, is a linear operator on $T_{\pi(J)} M$ whose value at $\xi_{\pi(J)}$ is zero.

Take a point $p \in M$. According to Proposition 3. $N_{1}^{(1)}\left(X^{h}, \xi^{h}\right)_{J}=0$ for $J \in \mathcal{C}_{p}$ and $X \in T_{p} M$, if and only if for every $Z, T \in \mathcal{D}_{p}$ and $J \in \mathcal{C}_{p}$

$$
\begin{gathered}
\omega(R(X, \xi) J Z, T)+\omega(R(X, \xi) Z, J T) \\
-\omega(J X, \xi) J Z, J T)+\omega(R(J X, \xi) Z, T)=0 .
\end{gathered}
$$

This obviously holds for $X=\xi$, so assume that $X \in \mathcal{D}_{p}$. Let $e_{\alpha}, \alpha=1, \ldots, 2 n$, be a symplectic basis of $\mathcal{D}_{p}$. It is convenient to set

$$
R_{\alpha, \beta, \gamma}=\omega\left(R\left(e_{\alpha}, \xi\right) e_{\beta}, e_{\gamma}\right)
$$

Then

$$
R_{\alpha, \beta, \gamma}=R_{\alpha, \gamma, \beta}=R_{\beta, \alpha, \gamma}
$$

For $\lambda \in \mathbb{R}$, consider the symplectic basis $e_{i}^{\prime}=e_{i}, e_{i+n}^{\prime}=\lambda e_{i}+e_{i+n}$. Applying (27) for the complex structure $J^{\prime}$ corresponding to this basis and $X=e_{i}^{\prime}, Z=e_{j}^{\prime}, T=e_{k}^{\prime}$, we obtain

$$
\begin{gathered}
R_{i, j, k}=0, \\
R_{i, j, k+n}+R_{i, j+n, k}+R_{i+n, j, k}=0, R_{i, j+n, k+n}+R_{i+n, j, k+n}+R_{i+n, j+n, k}=0 .
\end{gathered}
$$

Consider also the symplectic basis $e_{i}^{\prime \prime}=e_{i}+\lambda e_{i+n}, e_{i+n}^{\prime \prime}=e_{i+n}$. Setting $X=$ $e_{i}^{\prime \prime}, Z=e_{j}^{\prime \prime}, T=e_{k}^{\prime \prime}$ in (27) and taking into account (29), we get

$$
R_{i+n, j+n, k+n}=0, \quad R_{i+n, j+n, k}=0, \quad R_{i, j, k+n}=0 .
$$

Now $R_{i, j+n, k}=R_{i, k, j+n}=0$ and $R_{i, j+n, k+n}=R_{j+n, i, k+n}=R_{j+n, k+n, i}=0$ by (28) and (30). Similarly, identities (28) and (30) imply $R_{i+n, j, k}=R_{i+n, j, k+n}=0$. It follows that $R_{\alpha, \beta, \gamma}=0$ for every $\alpha, \beta, \gamma=1, \ldots, 2 n$. Therefore $\omega(R(X, \xi) Z, T)=0$ for every $Z, T \in \mathcal{D}$. This implies $R(X, \xi) Z=0$ since $R(X, \xi) Z \in \mathcal{D}$ and $\omega$ is non-degenerate on $\mathcal{D}$.

Conversely, if $R(X, \xi) Z=0$ for every $Z$, identity (27) is obviously satisfied, so $N_{1}^{(1)}\left(X^{h}, \xi^{h}\right)=0, X \in T M$.

Next, we discuss the identity $N_{1}^{(1)}\left(X^{h}, Y^{h}\right)_{J}=0$ for $X, Y \in \mathcal{D}_{\pi(J)}$. It is convenient to introduce the operator $J^{-}=\frac{1}{2}(I d+i J)$ on the complexification $\mathcal{D}^{\mathbb{C}}$ of $\mathcal{D}$ and to extend $\omega$ to $\mathcal{D}^{\mathbb{C}}$ by complex bilinearity. Then, taking into account Proposition 3 it is easy to check that $\omega\left(N_{1}^{(1)}\left(X^{h}, Y^{h}\right)_{J}(Z), T\right)=0$ for $X, Y, Z, T \in \mathcal{D}_{\pi(J)}$, if and only if $R_{\mathcal{D}}\left(J^{-} X, J^{-} Y,, J^{-} Z, J^{-} T\right)=0$. The latter condition is equivalent to $R_{\mathcal{D}}$ being of Ricci type by [10, Lemma 1.1]. 
This proves the first part of the theorem.

In order to see that the structure $\left(\Phi_{1}, \xi^{h}, G_{t}\right)$ is not normal, we fix a point $p \in M$ and take a symplectic basis $E_{1}, \ldots, E_{2 n}$ of $\mathcal{D}_{p}$. Let $J$ be the complex structure on $\mathcal{D}_{p}$ for which $J E_{i}=E_{i+n}, i=1, \ldots, n$. Define a vertical vector $V_{1,2}$ of $\mathcal{C}$ at $J$ by formula (1). Then $V_{1,2} E_{1}=E_{2+n}$, so $N_{2}^{(1)}\left(E_{1}^{h}, V_{1,2}\right)_{J}=-2\left(E_{2}^{h}\right)_{J} \neq 0$, by Proposition 3 .

Remark 5. Note that, by Corollary 4 the condition $R(X, \xi) Y=0$ for every $X, Y$ means that the vector field $\xi^{h}$ on $\left(\mathcal{C}, G_{t}\right)$ is Killing.

\section{Almost $C R$-structures on contact twistor space}

In this section we shall show that the almost $C R$-structure $\left(\mathcal{E}, \Phi_{2} \mid \mathcal{E}\right)$ is not integrable, reproving in passing the integrability result of [10] for $\left(\mathcal{E}, \Phi_{1} \mid \mathcal{E}\right)$; here $\mathcal{E}$ is the bundle over $\mathcal{C}$ whose fibre at a point $J \in \mathcal{C}$ is the space $\mathcal{V}_{J} \oplus\left\{X_{J}^{h}: X \in\right.$ $\left.\mathcal{D}_{\pi(J)}\right\}=\left(\mathbb{R} \xi_{\pi(J)}\right)^{\perp}$, the orthogonal complement being with respect to the metric $G_{t}$.

Recall that an almost Cauchy-Riemann $(C R)$ structure on a manifold $N$ is a pair $(\mathcal{E}, \Phi)$ of a subbundle $\mathcal{E}$ of the tangent bundle $T N$ and an almost complex structure $\Phi$ of the bundle $\mathcal{E}$. For any two sections $X, Y$ of $\mathcal{E}$, the value of $[X, Y] \bmod \mathcal{E}$ at a point $p \in N$ depends only on the values of $X$ and $Y$ at $p$, so we have a skewsymmetric bilinear form $\mathcal{L}: \mathcal{E} \times \mathcal{E} \rightarrow T N / \mathcal{E}$ defined by $\mathcal{L}(X, Y)=[X, Y] \bmod \mathcal{E}$ and called the Levi form of the $C R$-structure $(\mathcal{E}, \Phi)$. If the Levi form is $\Phi$-invariant, we can define the Nijenhuis tensor of the $C R$-structure $(\mathcal{E}, \Phi)$ by

$$
N^{C R}(X, Y)=-[X, Y]+[\Phi X, \Phi Y]-\Phi([\Phi X, Y]+[X, \Phi Y]) .
$$

The value of this tensor at a point $p \in N$ lies in $\mathcal{E}$ and depends only on the values of the sections $X, Y$ at $p$. An almost $C R$-structure is said to be integrable if its Levi form is $\Phi$-invariant and the Nijenhuis tensor vanishes. Let $\mathcal{E}^{\mathbb{C}}=\mathcal{E}^{1,0} \oplus \mathcal{E}^{0,1}$ be the decomposition of the complexification of $\mathcal{E}$ into $(1,0)$ and $(0,1)$ parts with respect to $\Phi$. If the $C R$-structure $(\mathcal{E}, \Phi)$ is integrable, then the bundle $\mathcal{E}^{1,0}$ satisfies the following two conditions:

$$
\mathcal{E}^{1,0} \cap \overline{\mathcal{E}^{1,0}}=0,\left[\Gamma\left(\mathcal{E}^{1,0}\right), \Gamma\left(\mathcal{E}^{1,0}\right)\right] \subset \Gamma\left(\mathcal{E}^{1,0}\right)
$$

where $\Gamma\left(\mathcal{E}^{1,0}\right)$ stands for the space of smooth sections of $\mathcal{E}^{1,0}$. Conversely, suppose we are given a complex subbundle $B$ of the complexified tangent bundle $T^{\mathbb{C}} N$ such that $B \cap \bar{B}=0$ and $[\Gamma(B), \Gamma(B)] \subset \Gamma(B)$ (many authors call a bundle with these properties " $C R$-structure"). Set $\mathcal{E}=\{X \in T N: X=Z+\bar{Z}$ for some (unique) $Z \in$ $B\}$ and put $\Phi X=-2 \operatorname{Im} Z$ for $X \in \mathcal{E}$. Then $(\mathcal{E}, \Phi)$ is an integrable $C R$-structure such that $\mathcal{E}^{1,0}=B$.

Let $\mathcal{L}_{k}$ be the Levi form of the almost $C R$-structure $\left(\mathcal{E}, \Phi_{k}\right), k=1,2$.

Lemma 8. Let $J \in \mathcal{C}, X, Y \in \mathcal{D}_{\pi(J)}$ and $U, V \in \mathcal{V}_{J}$. Then

$$
\mathcal{L}_{k}\left(X_{J}^{h}, Y_{J}^{h}\right)=-\omega(X, Y), \quad \mathcal{L}_{k}(U, V)=0, \quad \mathcal{L}_{k}\left(X^{h}, V\right)=0 .
$$

Proof. Extend $X$ and $Y$ to sections of $\mathcal{D}$ near the point $p=\pi(J)$ such that $\left.\nabla X\right|_{p}=$ $\left.\nabla Y\right|_{p}=0$. Then, by Lemma 5 .

$$
\mathcal{L}_{k}\left(X_{J}^{h}, Y_{J}^{h}\right)=G_{t}\left(\left[X^{h}, Y^{h}\right], \xi^{h}\right)_{J}=G_{t}\left(D_{X^{h}} Y^{h}-D_{Y^{h}} X^{h}, \xi^{h}\right)_{J}=-\omega_{p}(X, Y) .
$$

Extend $U, V$ to vertical vector fields. The vector fields $[U, V]$ and $\left[X^{h}, V\right]$ are vertical, hence $\mathcal{L}_{k}(U, V)=\mathcal{L}_{k}\left(X^{h}, V\right)=0$. 
Corollary 5. The Levy form $\mathcal{L}_{k}$ is $\Phi_{k}$-invariant, $k=1,2$.

Denote the Nijenhuis tensor of the $C R$-structure $\left(\mathcal{E}, \Phi_{k}\right)$ by $N_{k}^{C R}$.

Proposition 4. If $P, Q \in \mathcal{E}$, then $N_{k}^{C R}(P, Q)=N_{k}^{(1)}(P, Q)$.

Proof. Let $J \in \mathcal{C}$ and let $X, Y$ be sections of $\mathcal{D}$ near the point $p=\pi(J)$. Using Lemma 4 and identity (9), we easily see that

$$
\begin{gathered}
\mathcal{H} N_{k}^{C R}\left(X^{h}, Y^{h}\right)_{J}= \\
\left(-S_{p}\left(\nabla_{X} S\right)_{p}(Y)+S_{p}\left(\nabla_{Y} S\right)_{p}(X)+\left(\nabla_{S X} S\right)_{p}(Y)-\left(\nabla_{S Y} S\right)_{p}(X)\right)_{J}^{h} \\
+\left[\omega\left(X_{p}, Y_{p}\right)-\omega\left(S X_{p}, S Y_{p}\right)\right] \xi_{J}^{h} .
\end{gathered}
$$

Therefore,

$$
\mathcal{H} N_{k}^{C R}\left(X^{h}, Y^{h}\right)_{J}=0=\mathcal{H} N_{k}^{(1)}\left(X^{h}, Y^{h}\right)_{J}
$$

by Proposition 3. Lemma 4implies also that $\mathcal{V} N_{k}^{C R}\left(X^{h}, Y^{h}\right)_{J}=\mathcal{V} N_{k}^{(1)}\left(X^{h}, Y^{h}\right)_{J}$. It follows from Lemma 3 and Proposition 3 that $N_{k}^{C R}\left(X^{h}, U\right)_{J}=N_{k}^{(1)}\left(X^{h}, U\right)_{J}$ for every $U \in \mathcal{V}_{J}$. Finally, if $U, V \in \mathcal{V}_{J}$, then $N_{k}^{C R}(P, Q)=N_{k}^{(1)}(P, Q)=0$ since $\Phi_{k}$ is a complex structure on the fibres of $\mathcal{C}$.

Proposition 4 and the proof of Theorem 1 give the following.

Theorem 2. (i) ([10]) The almost $C R$ structure $\left(\mathcal{E}, \Phi_{1}\right)$ is integrable if and only if the tensor $R_{\mathcal{D}}$ is of Ricci type.

(ii) The almost $C R$ structure $\left(\mathcal{E}, \Phi_{2}\right)$ is never integrable.

\section{EXAMPLES}

Example 1. Let $E_{1}, E_{2}, E_{3}$ be left-invariant vector fields on the group $S O(3)$ such that

$$
\left[E_{1}, E_{2}\right]=E_{3}, \quad\left[E_{2}, E_{3}\right]=E_{1}, \quad\left[E_{3}, E_{1}\right]=E_{2} .
$$

If $E_{1}^{*}, E_{2}^{*}, E_{3}^{*}$ is the dual frame, set $\alpha=-E_{3}^{*}$. Then

$$
d \alpha\left(E_{1}, E_{2}\right)=1, \quad d \alpha\left(E_{1}, E_{3}\right)=d \alpha\left(E_{2}, E_{3}\right)=0 .
$$

Thus $\alpha$ is a contact form on $S O(3)$ with contact distribution $\mathcal{D}=\operatorname{span}\left\{E_{1}, E_{2}\right\}$ and Reeb vector field $\xi=-E_{3}$.

It is easy to check that for every contact connection the tensor $R_{\mathcal{D}}$ is of Ricci type.

A simple example of a contact connection $\nabla$ can be found setting

$$
\nabla_{E_{i}} E_{1}=a_{i} E_{1}+b_{i} E_{2}, \quad \nabla_{E_{i}} E_{2}=c_{i} E_{1}+d_{i} E_{2}, \quad i=1,2,
$$

where $a_{i}, b_{i}, c_{i}, d_{i} \in \mathbb{R}$. Then the identities

$$
\left(\nabla_{E_{1}} d \alpha\right)\left(E_{1}, E_{2}\right)=\left(\nabla_{E_{2}} d \alpha\right)\left(E_{1}, E_{2}\right)=0
$$

are equivalent to

$$
a_{1}=-d_{1}, \quad a_{2}=-d_{2} .
$$

We have

$$
\left[E_{1}, E_{2}\right]=\nabla_{E_{1}} E_{2}-\nabla_{E_{2}} E_{1}+\alpha\left(\left[E_{1}, E_{2}\right]\right) \xi, \quad i, j=1,2,
$$

if and only if

$$
a_{2}=c_{1}, \quad b_{2}=d_{1} .
$$


Suppose that identities (31) and (32) are satisfied and set $\nabla_{\xi} E_{i}=\left[\xi, E_{i}\right], i=1,2$, and $\nabla \xi=0$. Then $\nabla$ is a contact connection for which

$$
\begin{gathered}
R\left(E_{1}, \xi\right) E_{1}=\left(2 a_{2}+b_{1}\right) E_{1}+3 d_{1} E_{2}, \\
R\left(E_{1}, \xi\right) E_{2}=\left(c_{2}+2 d_{1}\right) E_{1}-\left(b_{1}-2 d_{2}\right) E_{2} \\
R\left(E_{2}, \xi\right) E_{2}=3 d_{2} E_{1}-\left(2 d_{1}+c_{2}\right) E_{2} .
\end{gathered}
$$

Thus $R(X, \xi) Y=0$ for $X, Y \in \mathcal{D}$ if and only if $a_{i}=b_{i}=c_{i}=d_{i}=0, i=1,2$.

Example 2. Let $G$ be the simply connected 5 -dimensional Lie group with Lie algebra generated by left-invariant vector fields $E_{1}, \ldots, E_{5}$ whose non-zero Lie brackets are

$$
\left[E_{2}, E_{3}\right]=E_{1}, \quad\left[E_{2}, E_{5}\right]=E_{2}, \quad\left[E_{3}, E_{5}\right]=-E_{3}, \quad\left[E_{4}, E_{5}\right]=E_{1} .
$$

One can easily see that the group $G$ is solvable. If $E_{1}^{*}, \ldots, E_{5}^{*}$ is the dual frame of left-invariant 1-forms, $E_{1}^{*}$ is a contact form on $G$. It is shown in 3 that $G$ admits a lattice $\Gamma$ such that the quotient $G / \Gamma$ is compact (recall that such a lattice is called uniform). In fact, $G$ is one of the groups in the list, obtained in [3], of all simply connected solvable 5-dimensional Lie groups admitting a left-invariant contact form and a uniform lattice.

Let $s \neq 0$ be a real number. Then $\alpha=s E_{1}^{*}+E_{4}^{*}$ is a contact form on $G$ with contact distribution $\mathcal{D}=\operatorname{span}\left\{E_{2}, E_{3}, E_{4}-\frac{1}{s} E_{1}, A_{5}\right\}$ and Reeb field $\xi=\frac{1}{s} E_{1}$. It is convenient to set

$$
A_{1}=E_{2}, \quad A_{2}=E_{3}, \quad A_{3}=E_{4}-\frac{1}{s} E_{1}, \quad A_{4}=E_{5}, \quad A_{5}=\xi .
$$

We have the following table for the non-zero Lie brackets of the vector fields $A_{1}, \ldots, A_{5}$

$$
\left[A_{1}, A_{2}\right]=s A_{5}, \quad\left[A_{1}, A_{4}\right]=A_{1}, \quad\left[A_{2}, A_{4}\right]=-A_{2}, \quad\left[A_{3}, A_{4}\right]=s A_{5} .
$$

The only non-zero values of the form $\omega=d \alpha$ are

$$
\omega\left(A_{1}, A_{2}\right)=\omega\left(A_{3}, A_{4}\right)=-s .
$$

Let $\nabla^{\prime}$ be the connection on $G$ for which

$$
\begin{gathered}
\nabla_{A_{i}}^{\prime} A_{j}=\frac{1}{2}\left(\left[A_{i}, A_{j}\right]-\alpha\left(\left[A_{i}, A_{j}\right]\right) \xi\right), \quad i, j=1, \ldots, 4, \\
\nabla_{A_{5}}^{\prime} A_{i}=\left[A_{5}, A_{i}\right], \quad i=1, \ldots, 4, \quad \nabla^{\prime} A_{5}=0 .
\end{gathered}
$$

This connection satisfies all conditions in the definition of a contact connection except the condition (8) (for example, $\left(\nabla_{A_{1}}^{\prime} \omega\right)\left(A_{2}, A_{4}\right)=-\frac{s}{2} \neq 0$ ). To get a contact connection we follow the procedure used in the proof of [10, Theorem 2.5]. If $\mathcal{N}$ is the tensor on $\mathcal{D}$ defined by

$$
\omega(\mathcal{N}(X, Y), Z)=\left(\nabla_{X}^{\prime} \omega\right)(Y, Z), \quad X, Y, Z \in \mathcal{D},
$$

set

$$
\begin{gathered}
\widetilde{\nabla}_{X} Y=\nabla_{X}^{\prime} Y+\frac{1}{3} \mathcal{N}(X, Y)+\frac{1}{3} \mathcal{N}(Y, X) \text { for } X, Y \in \mathcal{D}, \\
\widetilde{\nabla}_{\xi} X=[\xi, X] \text { for } X \in \mathcal{D}, \quad \widetilde{\nabla} \xi=0 .
\end{gathered}
$$

Then $\widetilde{\nabla}$ is a contact connection. 
In our case, the non-zero values of $\mathcal{N}$ are

$$
\begin{gathered}
\mathcal{N}\left(A_{1}, A_{2}\right)=\mathcal{N}\left(A_{2}, A_{1}\right)=\frac{1}{2} A_{3}, \quad \mathcal{N}\left(A_{1}, A_{4}\right)=-\frac{1}{2} A_{1}, \\
\mathcal{N}\left(A_{2}, A_{4}\right)=\frac{1}{2} A_{2} .
\end{gathered}
$$

Thus we have the following table for the connection $\widetilde{\nabla}$

$$
\begin{gathered}
\widetilde{\nabla}_{A_{1}} A_{2}=\frac{1}{3} A_{3}, \quad \widetilde{\nabla}_{A_{1}} A_{4}=\frac{1}{3} A_{1}, \quad \widetilde{\nabla}_{A_{2}} A_{1}=\frac{1}{3} A_{3}, \quad \widetilde{\nabla}_{A_{2}} A_{4}=-\frac{1}{3} A_{2} \\
\widetilde{\nabla}_{A_{4}} E_{1}=-\frac{2}{3} A_{1}, \quad \widetilde{\nabla}_{A_{4}} E_{2}=\frac{2}{3} A_{2}
\end{gathered}
$$

and all other $\widetilde{\nabla}_{A_{i}} A_{j}$ vanish.

The tensor $R_{\mathcal{D}}$ for this connection is not of Ricci type (identity (4) with $P=\sigma$ is not satisfied for $X=A_{1}, Y=A_{4}, Z=A_{4}, U=A_{4}$ ). According to [10, Theorem 2.5 ] every contact connection $\widetilde{\nabla}$ is of the form

$$
\nabla_{X} Y=\widetilde{\nabla}_{X} Y+S(X, Y)
$$

where $S(X, Y)$ is a tensor with the following properties:

(a) $S(X, Y)$ takes its values in $\mathcal{D}$;

(b) $S(X, \xi)=S(\xi, X)=0$ for every $X$;

(c) $S(X, Y)=S(Y, X)$;

(d) $\quad \omega(S(X, Y), Z)$ is a symmetric 3-tensor.

In order to find a tensor $S$ such that the curvature $R_{\mathcal{D}}$ of the corresponding connection $\nabla$ is of Ricci type, we have used a computer computation. This suggested the following simple choice of $S$

$$
\begin{gathered}
S\left(A_{1}, A_{2}\right)=S\left(A_{2}, A_{1}\right)=-\frac{1}{3} A_{3}, \quad S\left(A_{1}, A_{4}\right)=S\left(A_{4}, A_{1}\right)=-\frac{1}{3} A_{1}, \\
S\left(A_{2}, A_{4}\right)=S\left(A_{4}, A_{2}\right)=\frac{1}{3} A_{2},
\end{gathered}
$$

all other $S\left(A_{i}, A_{j}\right)=0$. Under that choice of $S$ we get a flat contact connection $\nabla$ for which

$$
\nabla_{A_{4}} A_{1}=-A_{1}, \quad \nabla_{A_{4}} A_{2}=A_{2}, \quad \nabla_{A_{i}} A_{j}=0 \text { for }(i, j) \neq(4,1),(4,2) .
$$

Example 3. As in [2, Example 2], consider a 5-dimensional Lie group $G$ with Lie algebra generated by left-invariant vector fields $E_{0}, E_{1}, \ldots, E_{4}$ whose non-zero Lie brackets are

$$
\begin{gathered}
{\left[E_{0}, E_{1}\right]=-E_{1}, \quad\left[E_{0}, E_{2}\right]=E_{2}, \quad\left[E_{1}, E_{2}\right]=E_{3},} \\
{\left[E_{1}, E_{4}\right]=-E_{1}, \quad\left[E_{3}, E_{4}\right]=-E_{3}}
\end{gathered}
$$

Let $s \neq 0$ be a real number. If $E_{0}^{*}, E_{1}^{*}, \ldots, E_{4}^{*}$ is the dual frame of left-invariant 1-forms, $\alpha=E_{3}^{*}+s E_{0}^{*}$ is a contact form on $G$ with contact distribution $\mathcal{D}=$ $\operatorname{span}\left\{E_{1}, E_{2}, E_{4}, s E_{3}-E_{0}\right\}$ and Reeb field $\xi=\frac{1}{s} E_{0}$. Set

$$
A_{1}=E_{1}, \quad A_{2}=E_{2}, \quad A_{3}=E_{4}, \quad A_{4}=s E_{3}-E_{0}, \quad A_{5}=\xi
$$

Considerations similar to that in the preceding example lead to the following nonflat contact connection $\nabla$ whose curvature $R$ satisfies the identity $R(X, \xi) Y=0$ 
for every $X, Y$ and the tensor $R_{\mathcal{D}}$ is of Ricci type (thus, the almost contact metric structure $\left(\Phi_{1}, \xi^{h}, G_{t}\right)$ is normal):

$$
\begin{gathered}
\nabla_{A_{1}} A_{2}=\frac{1}{2 s} A_{4}, \quad \nabla_{A_{1}} A_{3}=-\frac{1}{2} A_{1}, \\
\nabla_{A_{2}} A_{1}=-\frac{1}{2 s} A_{4}, \quad \nabla_{A_{2}} A_{3}=-\frac{1}{2} A_{2}, \\
\nabla_{A_{3}} A_{1}=\frac{1}{2 s} A_{1}, \quad \nabla_{A_{3}} A_{2}=-\frac{1}{2} A_{2}, \quad \nabla_{A_{3}} A_{4}=-2 A_{3}, \\
\nabla_{A_{4}} A_{1}=A_{1}, \nabla_{A_{4}} A_{2}=-A_{2}, \nabla_{A_{4}} A_{3}=-2 A_{3}-A_{4}, \nabla_{A_{4}} A_{4}=8 A_{3}+2 A_{4}, \\
\nabla_{A_{5}} A_{1}=-\frac{1}{s} A_{1}, \quad \nabla_{A_{5}} A_{2}=\frac{1}{s} A_{2}, \\
\text { all other } \nabla_{A_{i}} A_{j}=0 .
\end{gathered}
$$

The tensor $R_{\mathcal{D}}$ of the following non-flat contact connection is of Ricci type, while $R(X, \xi) Y$ is not identically zero, so the almost $C R$ structure $\left(\mathcal{E}, \Phi_{1}\right)$ is integrable but the almost contact metric structure $\left(\Phi_{1}, \xi^{h}, G_{t}\right)$ is not normal.

$$
\begin{gathered}
\nabla_{A_{1}} A_{2}=-\frac{1}{3 s} A_{3}+\frac{2}{3 s} E_{4}, \quad \nabla_{A_{1}} A_{3}=-\frac{2}{3} A_{1}, \quad \nabla_{A_{1}} A_{4}=-\frac{1}{3} E_{1}, \\
\nabla_{A_{2}} A_{1}=-\frac{1}{3 s} A_{3}-\frac{1}{3 s} A_{4}, \quad \nabla_{A_{2}} A_{3}=-\frac{1}{2} A_{2}, \quad \nabla_{A_{2}} A_{4}=\frac{1}{3} A_{2}, \\
\nabla_{A_{3}} A_{1}=\frac{1}{3} A_{1}, \quad \nabla_{A_{3}} A_{2}=-\frac{1}{3} A_{2}, \quad \nabla_{A_{3}} A_{3}=-\frac{1}{3} A_{3}, \nabla_{A_{3}} A_{4}=\frac{1}{3} A_{3} \\
\nabla_{A_{4}} A_{1}=\frac{2}{3} A_{1}, \quad \nabla_{A_{4}} A_{2}=-\frac{2}{3} A_{2}, \quad \nabla_{A_{4}} A_{3}=-\frac{2}{3} A_{4}, \\
\nabla_{A_{5}} A_{1}=-\frac{1}{s} A_{1}, \quad \nabla_{A_{5}} A_{2}=\frac{1}{s} A_{2}, \\
\text { all other } \nabla_{A_{i}} A_{j}=0 .
\end{gathered}
$$

Finally, note that the Lie algebra $\mathfrak{g}=\operatorname{span}\left\{E_{0}, \ldots, E_{4}\right\}$ of the group $G$ is solvable. It is not unimodular ( Trace $a d_{E_{4}}=2$ ), hence, by a result of Milnor [6], the group $G$ does not possess a discrete subgroup $\Gamma$ such that the quotient $G / \Gamma$ is compact.

\section{REFERENCES}

[1] D.E. Blair, Riemannian geometry of contact and symplectic manifolds, Progress in Mathematics, vol. 203, Birkhäuser, Boston Basel Berlin, 2002.

[2] A. Diatta, Left invariant contact structures on Lie groups, Diff. Geom. Appl. 26 (2008), 544-552.

[3] A. Diatta, B. Foreman, Lattices in contact Lie groups and 5-dimensional contact solvmanifold, Kodai Math. J. 38 (2015), 228-248.

[4] P. Liberman, Sur le problème d'èquivalence de certaines structures infinitèsimals, Ann. Mat. Pure Appl. 36 (1954), 27-120.

[5] D. McDuff, D. Salamon, Introduction to symplectic topology, Clarendon Press, Oxford, 1998.

[6] J. Milnor, Curvatures of left invariant metrics on Lie groups, Adv. Math. 21 (1976), 293329.

[7] C.L. Siegel, Symplectic geometry, Academic Press, New York, 1964.

[8] I. Vaisman, Symplectic curvature tensors, Monatshefte für Mathematik 100 (1985), 299327.

[9] I. Vaisman, Symplectic twistor spaces. J. Geom. Phys. 3 (1986), 507-524. 
[10] L. Vezzoni, Connections on contact manifolds and contact twistor spaces, Israel J. Math. 178 (2010), 253-267.

Institute of Mathematics and Informatics, Bulgarian Academy of Sciences, Acad. G.Bonchev St. Bl.8, 1113 Sofia, Bulgaria,

AND

University of Structural Engineering and Architecture "L.Karavelov", 175 SuhodolSKa St., 1373 Sofia, Bulgaria

E-mail address: jtd@math.bas.bg

Department of Mathematics and Computer Science, Eastern Connecticut State UniVERsity, Willimantic, CT 06226, USA

E-mail address: yankovc@easternct.edu 